\title{
Interactional features of a paper-based monitoring system
}

\author{
HANS TAP
}

Work practice laboratory, Blekinge Institute of Technology, PO Box 520, SE-372

25 Ronneby, Sweden

Hans.Tap@bth.se

Fax: +46 45712679

Tel: +46457385567

\begin{abstract}
Traditional desktop computers have a tendency to drag users to the desk and away from their object of work. Researchers working within fields covered by the term 'tangible computing' are trying to find ways to integrate computer technology in a more radical way and minimise gravitation to a desk. In this paper we argue for different interactional features that are connected to a design idea for monitoring treatment sessions of remote hemodialysis. We discuss interactional features with a non-traditional computer interface in a highly mobile work environment.
\end{abstract}

Keywords: Interactional features, tangible interaction

\section{Introduction}

One aspect when designing technology with visions like Ubiquitous Computing in mind is how to make interaction fit seamlessly into the kinds of everyday activity in which the technology is to be used. This paper will present a design concept for the purpose of remote haemodialysis monitoring with a focus on the interactional features that enable the design to fit nicely into the work practice. The design concept suggests a paper-based solution, but it is not our intention to suggest that this would be the single best solution. The idea presented here should be seen as a tool for discussing interactional features. These features can probably also be realised by implementing other design suggestions too, although we do find the paper-based version to be a rather convincing one.

The work presented is connected to a joint project between Blekinge Institute of Technology, a local hospital (Blekingesjukhuset) and Baxter Healthcare Corporation. The overall idea of the project was to enable the hospitalised haemodialysis patients to stay in a non-hospitalised environment where they can 
take greater - or at least more practical - responsibility for their treatment. Instead of having a dialysis nurse doing most of the work and being present throughout the treatment, the patients themselves are to do the work themselves. The goal is governed by both economic and medical factors. The dialysis departments need to keep their costs down, and with the continuous increase in the number of patients, this is hard to achieve. To let the patients take care of their own treatment to a greater extent is one way to handle the problem. Due to the limited number of beds at the hospitals the patients can 'only' have their treatment three times a week (for 4-5 hours per session). If the treatment can be more local and the patient do more of the work him-/herself, more frequent treatment becomes possible. Research has shown that patients show better clinical values if they can have dialysis treatment more frequently and for a longer time per session, as shown in, for example, Pierratos et al's. report of the success of nocturnal haemodialysis [1].

Due to the geographical distance between the patients and the nurses, we currently assume that there is good reason for the nurses to monitor the remote patients even though the patients themselves will take care of their own treatment. We assume there always will be problems and that patients will need a professional opinion; it might also be advantageous if the nurses can have an at-a-glance access to the quality of the remote patients' current treatment (especially initially, when the patient is inexperienced). Where a patient requests guidance, a continuous peripheral monitoring of the remote patient's state can be a convenient way of keeping medical staff updated and thereby enabling them to render fast assistance. Having a continuous view of the treatment progress can also be helpful when calibrating treatment. Minor anomalies in treatment values can easily be identified and spur the nurse to contact the patient.

We rely on an analysis of haemodialysis work practice and a design interest in the areas of ubiquitous computing and tangible computing as a base for our argumentation. What makes us especially interested in alternative computer interfaces such as those suggested by ubiquitous/tangible computing is how we perceive a connection to what is often noted in work practice studies. For example, Bowers et al. [2] who have studied a print industry shop floor show how the ecology of the workplace plays a significant role in cooperative aspects of 
work. Having co-workers and their machines in sight promotes awareness of other's work which in turn supports ad hoc cooperation (p. 56). Button and Sharrock [3] illustrate in a later paper the benefits of having artefacts available ata-glance. For example, by placing ‘job-flags’ which display miscellaneous job information into piles of printed paper, an at-a-glance revision of the production order is achieved, allowing people to move the jobs on. Harper and Hughes [4] describe how air traffic controllers arrange flight strips in a certain way and sometimes lift them slightly out of their racks as a reminder of a particular problem. It is not only concluded, however, that the strips can be arranged in certain ways, but that this also serves as an at-a-glance indication for other controllers of the current state of air traffic. Working with physical artefacts located in different parts of the working environment also makes it easier for people to keep track of what their co-workers are up to. Robinson [5] re-reports from a study in a French Nuclear Power Station where they were about to replace physical dials with computer displays that were easier to read for the individual operator. The downside, however, was that the operators were no longer able to see what other operators were doing. With the physical dials, it was easy to see which part of the system was of concern when someone went up to them. This feature would have disappeared with the new displays (which, incidentally, were never implemented partly for these reasons). In our own studies at dialysis departments we have, for instance, seen how the physical placement of alarm devices affects the ability to localise the origin of an alarm and how nurses orient themselves, depending on the design of the alarm system [6].

How people orient themselves in relation to artefacts, their physical environment, and one another in everyday work activities seems to create a special tension in relation to computer systems which rely on desktop computers as the base for computer interaction. We use the term desktop gravitation to describe how desktop computers force users to move up to the desk to carry out their work. This drags them away from their object of work. Fields such as ubiquitous computing, disappearing computing, and tangible computing are influential sources for inspiration, and often seem to suggest possibilities that could reduce the gravitational force of IT - although that particular aspect is not always mentioned. Further, we are aiming at achieving a seamless interaction (as also proposed by 
[7]) between the user and the technology by utilising the physical environment and physical objects.

Before we go into the details of the interactional features that are the main topic of this paper, we will first describe the relevant elements of the work practice in the haemodialysis department we have studied.

\section{Dialysis nurses' work practice}

Like many other work settings, the characteristic of medical work in hospitals is to a great extent mobile. At the dialysis departments we have studied, the mobility of the work is an obvious feature. Nurses are more or less constantly on the move and often switch between different work activities. Their work is frequently interrupted by questions from other nurses, phone calls, and alarms that require their attention.

Further, nurses' work can be characterised in part as opportunistic (in a positive sense). When the physician comes to the ward for his/her rounds, nurses take the opportunity to approach the physician with a variety of questions. One interesting part of these meetings is that the nurses often bring documentation (e.g. patient records) that is used as reference in the discussions.

In the dialysis department we have studied, the nurses are the most active ones when it comes to setting up the dialysis machines and making changes during treatment sessions [8]. The nurse is responsible for ensuring that the treatment meets certain goals prescribed, for example, by the physician. This can, for example, be the level of the blood rate and how much the patient should weigh once the sessions is ended ${ }^{1}$. There is also a general rule that the nurses should check up on their patients at least once an hour. The continuous monitoring by the nurses' keeps them constantly updated about the patient's condition and the ongoing treatment session. They stay "geared into the work", to borrow a phrase from Hughes et al. [9]. In a remote setting with the patient in another location, this possibility could be lost. The display used on the dialysis machine (which the

\footnotetext{
1 One important part of dialysis treatment is to remove the accumulated fluid in the patients, and the patients' weight before and after the treatment is used to measure the amount of fluid that has been removed.
} 
nurses rely on when working with local patients) often only shows the current values, and no historical data. But as already stated, nurses stay updated, i.e. keep a historical record of the values in mind, by being continuously engaged with a patient. They also take notes over some specific values in a special dialysis protocol. These values are also occasionally reflected upon during later treatment sessions (see [8] for more details). Although each nurse is responsible for certain patients, they regularly engage with other patients too in order to help the nurse responsible. When they intervene with other patients they communicate to the responsible nurse what has been done. This is usually done by looking them up, but also through signed notes in the dialysis protocol for each patient.

The understanding gained through our ethnographic study of the work at the dialysis department is an important base for our reasoning about new technology to support the work. The design idea presented and the proposed interactional features presented in the next sections have been developed with this in mind.

\section{Design concept}

There are many ways one could design a system for monitoring remote patients. What we were looking for was a system that not only visualised treatment data, but also had more interactive properties. We were, for example, looking at how to bring a reference to the remote patient/treatment to different locations. Further, we investigated ways of entering information dealt with outside a digital system back into it. This latter requirement caused us to discard many of our design ideas. Several suggestions produced interesting ways of visualising information but gave little scope for an easy or practical and seamless way of updating information back into the system. Many ideas would not fit the requirements suggested by the ethnographic study, i.e. showing consideration for the mobile character of nurses work, the way nurses bring reference material to the physician, that they stay 'geared into the work', continuously monitoring the patients, and the nurses' communication with one another when intervening with other nurses' patients.

\subsection{Paper-based monitoring system}

After working with several different design ideas, the idea that we decided to opt for was a paper-based monitoring system. Before we start to discuss the potential

\section{FIGURE 1}


interactional features that this design concept makes possible, we will first describe it briefly.

Basically every remote patient's treatment data should be displayed on one sheet of paper. As illustrated in the picture of a mock-up (figure 1), there are several small printers hanging on the wall. Each printer will be dedicated to one specific treatment session at a time. When a remote patient starts the treatment, one of the printers will become activated and treatment data will be continuously printed out on a sheet of paper. The length of the printed paper should show approximately how long the treatment has been in progress. An example of a printout is illustrated in figure 2.

\section{FIGURE 2}

Figure 2: An example of a paper strip showing treatment data

The paper printed on is not ordinary paper; it is based on the technique from Anoto. The paper has a pattern that can be recognised by the Anoto-pen, which allows you to track exactly where notations have been made on the paper ${ }^{2}$. This information can then be sent to a computer to be reused. We will describe the use of that feature later in this paper. The printer is also equipped with a sensor for knowing when someone has ripped of the paper. When the paper has been ripped off, a new copy will immediately be printed, and the following treatment data will be continued on the copy. Every time a new paper sheet is printed, an additional strip will be printed at the lower end of the paper. Also, if someone makes a notation with an Anoto-pen and chooses to save this, a special symbol will be printed (see fig 2). The next time the paper is reprinted, the symbol will have disappeared and the actual notation will be printed on the paper instead.

A barcode is printed at the bottom of each paper sheet. The barcode is an identifier which is connected to each patient's data. It is supposed to work as a way to connect easily to other services. By scanning the barcode on a computer containing patient records, the nurse can easily look up the patient in question.

\footnotetext{
${ }^{2}$ The Anoto pen has a small camera that registers the pen's movement when using it over a special kind of paper with a specific pattern. By recognising the pattern it is possible to determine exactly where on the paper notations have been made. This information is used to transform the stored coordinates into an image that can be reused and displayed on other devices. See www.anoto.com for more detailed information.
} 
There is no need to perform searches by entering social security numbers. One could also imagine that the barcode is scanned with a device connected to the telephone system which would minimize the need to look up the patient's phone number and so on. One reason for choosing barcodes is that their function is widely recognised. People know that they are supposed to be scanned and how this is done.

\section{Interactional features}

As we know, paper has many qualities that make it extremely useable [10], and some have tried to use paper when designing new interactive tools [11] [12]. What can be done with paper is common knowledge to most of us. We know, for example, that it is possible to write and draw on it, that it is easy to fold, and that it disappears when you put it on fire. Also, in cooperative situations paper has qualitative benefits. Luff and Heath [13] use the term micro-mobility to describe a frequently ignored feature of technology, i.e. "the way in which an artefact can be mobilised and manipulated for various purposes around a relatively circumscribed, or 'at hand', domain” (p. 306). They point specifically to paper's high 'ecological dexterity' as compared to conventional computer systems (both desktop and portable). This feature alone makes design based on paper fascinating. The rationale in our case is that we wish to utilise the highly mobile characteristic of nurses' work described earlier and make our design fit into their way of working.

\subsection{Zooming by walking}

The fact that nurses move from room to room and in the corridor can be used as a resource for designing interactional features. In our design suggestion we consciously utilise the distance between the nurse and the artefacts. As shown in figure 2, there are different patterns and figures printed on the paper. The patterns are intended to show graphically the development of different treatment values. These patterns make it possible to see at-a-glance and from several meters away how the values have changed over time. By moving closer to the paper sheets you can obtain precise treatment data by reading the printed numbers - what we call 'zooming by walking'. Zooming by walking is typical of the nurses' everyday work and integrated in many of their mundane activities. For example, when an 
alarm goes off they first decode figures on a display stating, for example, from which room and dialysis machine the alarm comes from [5]. Then they walk into that particular room towards the dialysis machine causing the alarm. Inside the room it is sometimes (depending on what kind of alarm it is) possible to see, from several meters, indications on the visual display of the kind of alarm it is. When moving closer nurses can further inspect the figures displayed on the dialysis machine's display. To move up close in order to see more detailed information is something we all do on occasions and it is of course not a special feature of dialysis nurses' work. What we are doing is to consider consciously this aspect of interaction with artefacts when designing our monitoring system.

There is another reason too for taking this path. By only showing abstract visualisations of treatment data from a distance, we let the nurses decide themselves when they want more detailed information. It is a way of keeping the monitoring system on the periphery and lets the nurses pull it into the foreground when they choose to (typically by moving up closer to the paper sheet).

\subsection{Traces of action}

When placing a system in the corridor the treatment data becomes more accessible for all to see as compared to placing individual dialysis machines in the wards thereby requiring that you enter the particular ward. Due to the way the staff is organised, i.e. each nurse is responsible for two patients, and the fact that patients are located in different rooms, individual patient's treatment data is less accessible than if it were placed in the corridor where more people pass by more regularly. The increased availability of the treatment data that our design suggests could potentially lead to more people being engaged with the data in different ways (if so only by a mere glance). One potential issue could be that several people engage individually with the treatment for the same reason, i.e. conducting the same work. As a way of limiting these instances, we wish to offer the possibility to indicate that someone has already engaged with the patient (or at least the paper strip). We want to leave traces of actions for others to see - an asynchronous account of actions. 
Our suggestion consists of two parts. The first is to print a small stripe at the bottom of the paper sheet every time the paper is re-printed (happens when the paper is ripped of from the printer). The stripes do not say anything about the reason or motivation for why someone has ripped of the paper. It indicates that for some unknown reason someone has ripped of the paper, but also suggests, however, that someone has been engaged with the treatment. Our assumption is that this indication will let the nurses draw conclusions as to what is likely to have been done. Secondly, it will also be possible to make annotations on the paper using such technology as Anoto. When someone decides to save the annotations made by an Anoto-pen, by marking a specific part of the paper, a symbol will be printed on the paper currently in the printer. The symbol states that someone has made an annotation. A copy of the actual annotation will first be printed on the next paper sheet - after someone has ripped of the paper with the symbol.

\subsection{Mobile manipulation}

One could guess, based on current computer systems that are traditionally used, that a classic design for a monitoring system, in this case for remote patients, would consist of a computer screen and a desktop computer with which you interact using a mouse and keyboard. In order to inspect the status of the remote patients, the nurse would have to go up to the desk where the computer is located. If the nurse would like others to see the data and discuss it, it is likely that she would have to go and look for the other person and let them follow her back to the desk. One could assume that a print function exists to print out the treatment data. This could let the nurse bring the paper to the people she wants to discuss with instead of taking him/her to the computer, but she would most probably be needed to input notes from their discussion at the computer. Our aim is to limit this potentially high degree of desk gravitation, and utilise the fact that nurses are moving around in the dialysis department.

As briefly pointed out earlier, paper has benefits over traditional screens in the very local interaction between collocated persons. If the topic for discussions is fuelled with data presented on a screen, there is a need for both parties to locate themselves physically in front of it in order to see what is being referred to. If we compare with data that are presented on a piece of paper, it is often easy to make 
out what it is about even if you see the information upside down. Further, if there is a need to show the data in more detail, the paper is easily turned around and oriented towards the person of interest. This is often a cumbersome task to perform with traditional computer screens. One could imagine palm computers being used for this, but even they have problems with simultaneous viewing depending on the angle from which you look. There are, however, interesting attempts to design palm computers with an increased support for micro-mobility (although these words are not used). By equipping handheld devices with sensors, Hinckley et al indicate, for example, the possibility to tilt the device away from oneself, causing the content of the display to rotate 180 degrees so it is readable for someone on the opposite side [14].

\section{Concluding remarks}

The possibility to tear off the paper and bring it to wherever it is needed is one attempt to relieve gravitational force to the desk. The ability to input annotations on the paper and for these to be transferred to its digital counterpart further weaken desk gravitation. The staff can, at their current location, input data without needing to go back to a computer.

It is clear that our solution also has gravitational force as the nurses must approach the printer on the wall to get hold of the paper. One could argue that it would be better to equip every nurse with a palm computer in order to minimise gravitation towards a specific place. We believe, however, that some gravitation is actually desirable. The reason is visibility.

Imagine that someone is sitting in front of a traditional desktop computer. In order to see what the person is engaged in it is often necessary to move up behind the person and rather close to the screen. It might be possible from a distance to see that the person is using a word processor, but it can be hard to see which document the user is working with. If you cannot see the screen then it is hard to make out what the person is doing on the computer. It is hard to know the details 
of which object (application and its current content) the user is working on at a mere glance ${ }^{3}$.

As seen in the nuclear power plant example, gravitation can be desirable. The need for someone to locate herself physically at a specific place makes it possible for others to draw conclusions about what is going on. By placing our design in the corridor and making people move closer to it when interacting with it, we hope to provide the possibility for others to recognise at a glance what the person is doing. In our design you can, for example, see which paper strip that is occupying a nurse's attention and thus know which patient it is, and you can see if the paper is being ripped off, and based on where the nurse is going to, it is possible to some extent to foresee the coming action (did the nurse go into the video conference room, the lunch room where the physician is having a coffee, or to a desktop computer in the office?).

Further, there are also organisational issues that need to be considered. One needs to decide what the nurses' role will be towards the remote patient. What kind of responsibility will she have for the quality of the treatment? We assume the main responsibility will be in the hands of the patient since the nurses cannot control the treatment and physically help the patient. What can be important to think further about is what our design suggests for the nurses in relation to responsibility. Although they might not be responsible for the immediate safety of the remote patient, the monitoring system gives them information that in many ways affords responsibility. It is potentially possible to see that the treatment is not proceeding as it should, and that would be hard to disregard for any nurse even if it is not their concern formally. It is also possible that the more detailed information that is presented about the treatment, the more responsibility is afforded. If we imagine a situation where there is a light in the corridor showing the overall status of all remote patients, there would probably be fewer occasions for intervention. What the role of the nurses will be will influence the final details of the design.

\footnotetext{
${ }^{3}$ The reason for this is not only due to the physical design of screens, but also due to the amount of different applications that potentially can be run on a computer. Our design suggestion is more limited in terms of what it is used for - printing treatment data. So it is of course also easier to understand what someone is doing with a 'single function' artefact, than with one supporting 'multiple functions'.
} 
It is also important to point out that we are interested in the interactional aspects the design can provide. It is not our current concern to solve the problem of responsibility. What we want to stress is how our suggestion promotes 'visibility of work'; in particular, how the system through its use contributes to the accountability of the work situation. Here we use the ethnomethodological definition of accountability, i.e. "members' methods for making [...] activities visibly-rational-and-reportable-for-all-practical-purposes, i.e. 'accountable"' ([15] p. vii).

In this paper we have discussed how a paper-based display can be used and what the interactional strengths might be. We suggest a system that uses both the benefit of computer technology and people's way of interacting in their everyday activities by letting the human-computer interaction take a step away from the desktop computer.

\section{Acknowledgements}

I would specially like to thank Isa Hardemo and Jessica Svensson for participating in the development of different design ideas for remote heamodialysis monitoring that has lead to the ideas presented in this paper.

\section{References}

[1] Pierratos, A., M. Ouwendyk, R. Francoeur, S. Vas, D.S.C. Raj, A.-M. Ecclestone, V. Langos, \& R. Uldall (1998) Nocturnal Hemodialysis: Three-Year Experience, Journal of the American Society of Neprology

[2] Bowers, J., G. Button, \& W. Sharrock, (1995). Workflow From Within and Without: Technology and Cooperative Work on the Print Industry Shopfloor. Proceedings of the Fourth European Conference on Computer-Supported Cooperative Work. ECSCW '95. Y. S. a. K. S. Hans Marmolin. Dordrech/Boston/London, Kluwer Academic Publishers

[3] Button, G. and W. Sharrock (1997). The production of order and the order of production: Possibilities for distributed organisations, work and technology in the print industry. ECSCW '97. Proceedings of the Fifth European Conference on Computer-Supported Cooperative Work, 7-11 September 1997, Lancaster, U.K. J. A. Hughes, W. Prinz, T. Rodden and K. Schmidt. Dordrecht, Kluwer Academic Publishers

[4] Harper, R. H. R. and J. A. Hughes (1993). What a f-ing system! Send 'em all to the same place and then expect us to stop 'em hitting. Managing technology work in air traffic control. Technology in Working Order. Studies of work, interaction, and technology. G. Button. London and New York, Routledge: 127-144. 
[5] Robinson, M. (1993). Design for Unanticipated Use. Proceedings for ECSCW'93: Third European Conference on Computer Supported Cooperative Work, Milan, Italy, ACM Press.

[6] Sánchez Svensson, M. and H. Tap (2003). Alarms - Localisation, Orientation, and Recognition. International Journal of Human-Computer Interaction, Lawrence Erlbaum Associates, 15(1)

[7] Ishii, H. and B. Ullmer, (1997) Tangible Bits: Towards Seamless Interfaces between People, Bits and Atoms. in CHI 97, Conference on Human Factors in Computing Systems, (Atlanta, Georgia, USA

[8] Tap, H. (2002). Nurses' methods and their relation to design. Occasional papers from the Work Practice Laboratory, Department of Human Work Science and Media Technology, Blekinge Institute of Technology. 2:2002: 16 .

[9] Hughes, J., D. Randall, \& D. Shapiro (1993) From ethnographic record to system design. Some experiences from the field, Journal of CSCW, 3(1)

[10] Sellen, A. J. and R. H. R. Harper (2001). The myth of the Paperless Office, MIT Press.

[11] Rønby Pedersen, E., T. Sokoler, L. Nelson (2000). PaperButtons: Expanding a Tangible User Interface. Symposium on Designing Interactive Systems, Brooklyn, New York, ACM.

[12] Nelson, L., S. Ichimura, E. Rønby Pedersen, \& L. Adams (1999) Palette: a paper interface for giving presentationsCHI'99 (Pittsburg, Pennsylvania, USA, ACM Press).

[13] Luff, P. and C. Heath (1998). Mobility in Collaboration. Proceedings of the CSCW'98 conference. Seattle, ACM: 305-314.

[14] Hinckley, K., J. Pierce, M. Sinclair, \& E. Horvitz (2000) Sensing Techniques for Mobile InteractionUIST 2000 (Orlando, Florida, New York: ACM Press).

[15] Garfinkel, H. (1967) Studies in Ethnomethodology, Englewoods-Cliffs, New Yersey: Prentice-Hall. 